\title{
Characteristics of Resting-State Functional Connectivity in Older Adults after the PICMOR Intervention Program: A Preliminary Report
}

Hikaru Sugimoto ( $\square$ hikaru.sugimoto@riken.jp )

RIKEN https://orcid.org/0000-0003-1058-6714

Toshikazu Kawagoe

Rikkyo University

Mihoko Otake-Matsuura

RIKEN

\section{Research article}

Keywords: Intervention, Left inferior frontal gyrus, PICMOR, Resting-state functional MRI, Temporal pole, Verbal fluency

Posted Date: October 14th, 2020

DOI: https://doi.org/10.21203/rs.2.21637/v2

License: (a) (1) This work is licensed under a Creative Commons Attribution 4.0 International License. Read Full License

Version of Record: A version of this preprint was published on November 20th, 2020. See the published version at https://doi.org/10.1186/s12877-020-01892-2. 


\section{Abstract}

Background: The present study aimed to provide a basis for future research examining the neural mechanisms that underlie the beneficial effect of an intervention program, Photo-Integrated Conversation Moderated by Robots (PICMOR), on verbal fluency in older adults as identified in our previous randomized controlled trial. In this preliminary report, we conducted an additional experiment using resting-state functional magnetic resonance imaging (rsfMRI) after the intervention period. Specifically, we investigated the resting-state functional connectivity ( $\mathrm{rSFC}$ ) characteristics of the intervention group (INT) compared to the control group (CONT).

Methods: rsfMRI data were acquired from 31 and 30 participants in INT and CONT, respectively, after the intervention. In the analyses, two of the most important regions in verbal fluency, the left inferior and middle frontal gyri, were selected as seed regions, and the rsFCs were compared between groups. We also conducted regression analyses for rsFCs using the difference in individual phonemic verbal fluency task (PVFT) scores between the pre- and post-intervention periods (i.e., post- minus pre-intervention) as an independent variable.

Results: We found higher rSFC in INT than in CONT between the left inferior frontal gyrus as a seed region and the temporal pole and middle frontal gyrus. The rsFC strength between the left inferior frontal gyrus and temporal pole positively correlated with an increased PVFT score between the pre- and postintervention periods. In contrast, we found lower rsFC in INT than in CONT between the left middle frontal gyrus as a seed region and the posterior cingulate cortex, precuneus, and postcentral gyrus.

Conclusions: Our findings suggest that the beneficial intervention effect of PICMOR on verbal fluency is characterized by enhanced rsFC of the left inferior frontal gyrus with semantic and executive controlrelated regions and suppressed rsFC between the left middle frontal gyrus and posterior cortical midline structures. No definitive conclusions can be made because of a lack of rsfMRI data before the intervention. However, this pilot study provides the candidates for rsFCs, reflecting the beneficial effects of PICMOR on the brain network involved in verbal fluency.

Trial registration: The trial was retrospectively registered at the UMIN Clinical Trials Registry (UMIN000036667) (May 7th, 2019).

\section{Background}

Interest in the development of methods to prevent or delay dementia is growing rapidly [1]. Given the evidence of a relationship between cognitive function and social interaction [2], interventions that include social activities could be candidates for such methods. However, there is still little evidence regarding the effects of social activity-based interventions on cognitive function [3]. Toward addressing this gap, we have developed an intervention program, Photo-Integrated Conversation Moderated by Robots (PICMOR) [4]. The PICMOR offers a moderated group conversation context with robot-based facilitation.

Specifically, a robot encourages a participant to talk about their daily life, using photos they have 
prepared beforehand, and to answer questions from the other human participants. The other participants are required to listen carefully and ask the speaker questions. This program is partly characterized by enforced output, where the system and robot have been designed to directly and strongly encourage participants to talk about various topics for a certain length of time. To examine the effect of PICMOR on cognitive function in older adults, we conducted a randomized controlled trial (RCT) [4]. The main finding of our RCT was that there was a significantly larger increase in the phonemic verbal fluency task (PVFT) score, which is included in the Japanese version of the Montreal Cognitive Assessment [5], in the intervention group (INT) than in the control group (CONT) between the pre- and post-intervention periods. Therefore, it is reasonable to presume that this behavioral effect could be reflected in differences between the two groups in the brain network responsible for verbal fluency. To examine the differences between INT and CONT in the functional network of the brain regions involved in verbal fluency, we conducted an additional experiment after the intervention period using resting-state functional magnetic resonance imaging (rsfMRI), which has been associated with an individual's ability to perform cognitive tasks [6], including phonemic fluency ability [7].

In this preliminary report, we performed a seed-based functional connectivity analysis on the rsfMRI data [8], which were obtained after the intervention period from participants in our previous RCT study. In the analyses, we employed the left inferior and middle frontal gyri as seed regions, including a classical language-related region, called Broca's area [9-11]. These regions were selected because it has been suggested that they are the most important for verbal fluency. Indeed, previous neuropsychological studies have consistently shown that patients with lesions of the left frontal lobe have impaired phonemic verbal fluency [12-15]. In addition, previous neuroimaging meta-analyses have reported that the left inferior frontal gyrus and/or middle frontal gyrus consistently show significant activation during PVFTs $[10,11]$. These findings suggest that the left inferior and middle frontal gyri play pivotal roles in phonemic verbal fluency. Thus, we examined how the resting-state functional connectivity (rsFC) with these seed regions differed between INT and CONT, assuming that the differences between the groups were associated with the relatively large enhancement of the PVFT score in INT compared to CONT [4]. The differences between INT and CONT cannot be entirely attributed to the effect of PICMOR because we lack comparable rsfMRI data from the pre-intervention period. Therefore, no definitive conclusions can be made from this preliminary report. However, this study provides the candidates for rsFCs, reflecting the beneficial intervention effects of PICMOR on the brain network involved in verbal fluency.

\section{Methods}

\section{Participants}

Sixty-five healthy community-dwelling older adults, who participated in our previous RCT study (32 and 33 participants in INT and CONT, respectively) [4], were recruited in the present study. A total of sixty-one participants, including 31 and 30 participants in INT and CONT, respectively, took part in MRI scanning. MRI data from four RCT participants were not collected because one and one participant in INT and CONT, respectively, had claustrophobia, one participant in CONT was equipped with a heart pacemaker, 
and one participant in CONT declined to undergo the MRI scanning. All participants were right-handed and native Japanese-speaking individuals. We confirmed that there were no significant differences in age $(t[d f=59]=1.01, p=0.32), \operatorname{sex}\left(c^{2}[d f=1, \mathrm{n}=61]=0.15, p=0.70\right)$, and educational level $\left(c^{2}[d f=1, \mathrm{n}=\right.$ $61]=0.13, p=0.71$ ) between the two groups (educational level was binarized with a border of 13 years to categorize participants depending on whether they went to university or college; see Table 1).

Table 1 about here

\section{Intervention program}

The procedures used in the PICMOR intervention and control program are described in detail below. Both the intervention and control programs were based on a group conversation. The 65 people recruited from the Silver Human Resources Center were divided into 16 groups (eight and eight in INT and CONT, respectively), each with four members, except for one CONT, which had five members. The participants were required to participate in the group conversations once a week for 12 weeks. One of the major differences between the two programs was whether the programs were designed to train executive functions.

In the group conversation offered by PICMOR, a robot acted as the chair to lead the conversations and prompt one of the four members to speak about an event they had experienced in their daily life for 1 min. The topic was a predetermined subject that changed every week. During this period, the other three members of the group had to listen attentively so that they could ask questions during the discussion period. The 1 min talking period was repeated without a break, in which they talked about another event related to the topic (i.e., each participant was assigned a total of $2 \mathrm{~min}$ to talk). Following this, there was a 2 min discussion period for each event during which the speaker was required to answer questions raised by the other three members of the group. During the discussion periods, the robot automatically encouraged and stopped the participants' utterances to balance the amount of talking time allocated to each participant. For example, when the robot detected that one participant had spent less time talking than others, it directly prompted the participant to comment or ask a question. After the 2 min discussion periods, another member was assigned as the speaker. This procedure (i.e., the 1 min talking periods, followed by the 2 min discussion periods) was repeated for all members. There were two major reasons for using a robot and not a human as the chair. First, we could force the participants to make a speech during the predetermined time and finish their talk when their allocated time was over, and ensure that uncontrollable personal factors that can arise from a human chair, such as hesitation, were excluded. Time management by the robot made it possible to give each member an equally predetermined time (i.e., $1 \mathrm{~min}$ ) to talk about an event. Second, it would be challenging for a human chairperson to prompt and stop the conversations in real-time based on the talking time of each person.

In contrast, in the group conversations offered by the control program, four members were required to talk freely without any robotic facilitation or predetermined theme, similar to how they would converse in their daily life. As shown in Table 1, the variance of the amount of time spent talking in group conversations 
during the intervention period was smaller in INT than in CONT. In an F-test to compare the variances between the two groups, the null hypothesis (i.e., the true ratio of variance is 1 ) was rejected (ratio of variances $=17.79, p<0.01$ ). This suggests that our experimental manipulation by robotic moderation to balance the amount of talking time for each participant in INT was successful. We hypothesized that repeated training in group conversations in PICMOR compared to the control program would exercise executive functions, such as flexibility, planning, working memory, and response inhibition, given that the participants have to make a speech within a limited time (i.e., $1 \mathrm{~min}$ ), flexibly ask and answer questions, intentionally store and manipulate information to ask questions, and suppress the interruption of other members in a group conversation. Executive control and verbal abilities can be measured using the verbal fluency task [16], in which participants are required to produce as many words as possible beginning with a specific letter (e.g., / ka/ in Japanese) [5]. The number of correct unique words generated in $1 \mathrm{~min}$ is often used as a measure of performance. To successfully perform this task, participants must flexibly retrieve appropriate words along with the task rules, from their long-term memory (semantic memory), which most likely involves accessing their mental lexicon [16-18]. Successful task performance also requires them to keep previously produced words in their working memory so as to avoid repetition and suppress inappropriate words or task-irrelevant thoughts, which may involve executive control processes [16-18]. Given that the characteristics of the training demands in PICMOR involve exercising executive functions, it is reasonable to predict that the ability to produce words within a limited time could be enhanced in INT compared with CONT between the pre- and post-intervention periods. Consistent with this idea, a significantly larger improvement in the PVFT score was observed in INT than in CONT in our previous RCT study [4]. The beneficial intervention effect on verbal fluency could not be explained by the amount of talking time in group conversations during the intervention period, given that the talking time was significantly shorter in INT than in CONT $(t[d f=59]=4.21, p<0.01$, Cohen's $d=1.08$; see Table 1$)$.

\section{Data acquisition}

All MRI data were acquired with a Philips Achieva 3.0 MRI scanner, located in the Advanced Imaging Center Yaesu Clinic, Tokyo. Data were collected only after the intervention. During the MRI scanning, participants were equipped with a set of earplugs and headphones to reduce the effects of scanner noise and a belt with foam pads around their head to minimize head motion. There was no significant difference in the time period from the last day of the intervention period to the day of MRI data acquisition between INT (mean \pm SD $=9.67 \pm 0.76$ weeks) and CONT (mean \pm SD $=9.68 \pm 0.56$ weeks) $(t$ $[d f=59]=0.05, p=0.96)$.

First, three directional T1-weighted anatomical planes were scanned to localize the subsequent anatomical and functional images. Subsequently, anatomical structures were scanned using a highresolution T1-weighted image (repetition time $[\mathrm{TR}]=6.41 \mathrm{~ms}$, echo time $[\mathrm{TE}]=3.00 \mathrm{~ms}$, field of view [FOV] $=24.0 \mathrm{~cm} \times 24.0 \mathrm{~cm}$, matrix size $=256 \times 256$, slice thickness $/$ gap $=1.2 / 0 \mathrm{~mm}, 170$ sagittal slices) . Finally, resting-state functional images were scanned using a pulse sequence of gradient-echo echoplanar imaging, which is sensitive to blood oxygenation level-dependent (BOLD) contrasts (TR = $3000 \mathrm{ms,}$ 
$\mathrm{TE}=30 \mathrm{~ms}$, flip angle $=80$ degrees, $\mathrm{FOV}=24.0 \mathrm{~cm} \times 24.0 \mathrm{~cm}$, matrix size $=80 \times 80$, slice thickness $/ \mathrm{gap}=$ $4.0 / 0 \mathrm{~mm}, 35$ horizontal slices). All participants were instructed to remain awake with their eyes open and think of nothing during the entire rsfMRI scan $(10 \mathrm{~min})$. The rsfMRI run began with dummy scans that were discarded from further analyses.

\section{Data analysis}

All MRI data were analyzed using the CONN functional connectivity toolbox v.17.f (www.nitrc.org/projects/conn) [19] for Statistical Parametric Mapping 12 (SPM 12) (www.fil.ion.ucl.ac.uk/spm/), implemented in MATLAB. Resting-state functional images were preprocessed along the default pipeline in CONN. The images were realigned and corrected for slice timing. After the outlier detection, the functional and structural images were segmented and normalized to the Montreal Neurological Institute (MNI) space with a resolution of $2 \times 2 \times 2 \mathrm{~mm}^{3}$ voxels. These normalized functional images were spatially smoothed by a Gaussian kernel of $8 \mathrm{~mm}$ full-width at halfmaximum. Subsequently, temporal correction was performed using the component-based noise correction method [20]. In this step, five principal components were extracted from the white matter and cerebrospinal fluid regions. Along with six bulk motion parameters, the first-order derivative of each motion parameter, and the scrubbing parameter, the five principal components were regressed out from the signal of interest. The scrubbing parameter was provided using the Artifact Detection Tools in CONN that can detect outliers based on the variance of whole movements. A band-pass filter with a frequency window of $0.008-0.09 \mathrm{~Hz}$ and detrending were then applied to the data.

In the present study, we used seed-to-voxel analyses of the rsfMRI data. Seed regions were defined as spheres with a $5 \mathrm{~mm}$ radius, around $(-50,12,24),(-48,28,14),(-52,12,0),(-42,8,36),(-54,2,46)$, and $(-44,18,6)$ in the MNI coordinates, located in the left inferior and middle frontal gyri, based on a previous neuroimaging meta-analysis demonstrating that these regions consistently show significant activation during PVFTs [11]. The anatomical mask of these seed regions was created using MarsBaR (www.marsbar.sourceforge.net). In the individual-level analysis, the mean BOLD time course was extracted from each seed region, and correlation coefficients were calculated with the BOLD time course of each voxel, throughout the whole-brain. The coefficients were converted to normally distributed scores using Fisher's transformation. This procedure yielded individual rsFC maps for each seed region. In the group-level analysis, the rsFC maps identified in the first level analysis of INT and CONT were compared using two-sample t-tests. The models included age, sex, and educational level as covariates. In this second-level analysis, the threshold at the cluster level was corrected for whole-brain multiple comparisons (false discovery rate [FDR]; $p<0.05$ ). Given that we selected six seed regions for analysis, we also employed a stringent statistical significance in the two-sample $t$-tests. In this case, the height threshold was divided by the number of seed regions (FDR, $p<0.05 / 6$ ).

We also performed regression analyses for rsFCs using the raw scores of PVFT collected from all participants before and after the intervention. In this analysis, regions that showed significant 
correlations between the rsFCs with seed regions and a difference in the individual PVFT scores between the pre- and post-intervention periods (i.e., post- minus pre-intervention) as an independent variable were explored at the whole-brain level. This analysis enabled us to identify regions that modulated the increase in the score by interacting with the left inferior and middle frontal gyri as seed regions. Participants' age, sex, and educational level were also included as covariates in the analysis. The threshold at the cluster level was corrected for whole-brain multiple comparisons (FDR, $p<0.05$ ).

\section{Results}

In the present study, the strength of rsFC with the left inferior and middle frontal gyri as seed regions was compared between INT and CONT using two-sample t-tests. As illustrated in Fig. 1, significantly higher rsFC was identified in INT than in CONT between the left inferior frontal gyrus seed centered at $(-52,12$, $0)$ and the temporal pole, and between the left inferior frontal gyrus seed centered at $(-44,18,6)$ and the middle frontal gyrus (FDR, $p<0.05)$. In contrast, two-sample $t$-tests demonstrated that there was significantly lower rsFC in INT than in CONT between the left middle frontal gyrus seed centered at $(-42$, $8,6)$ and the posterior cingulate cortex, precuneus, and postcentral gyrus (FDR, $p<0.05)$. We did not identify any regions where rsFC was significantly different between INT and CONT with other seeds (FDR, $p<0.05$ ). Even when a stringent height threshold was used for whole-brain multiple comparisons, there

were rsFC values that remained significant, including the rsFCs between the left inferior frontal gyrus seed centered at $(-44,18,6)$ and the middle frontal gyrus, and between the left middle frontal gyrus seed centered at $(-42,8,6)$ and the posterior cingulate cortex (FDR, $p<0.05 / 6)$. The findings from the twosample $t$-tests are summarized in Table 2.

Fig. 1 \& Table 2 about here

For further analysis, we conducted regression analyses, using the difference in the PVFT scores between the pre- and post-intervention periods for all participants. The mean of the difference in the PVFT scores between the pre- and post-intervention was 0.82 (SD 3.51). We confirmed that there was a significant difference in the PVFT scores between the groups after the intervention $(t[d f=59]=3.16, p<0.01$, Cohen's $d=0.81)$ but not before the intervention $(t[d f=59]=0.91, p=0.36$, Cohen's $d=0.23$; see Table 1). The regression analyses showed that the increased PVFT score was significantly correlated with increased rsFC between the left inferior frontal gyrus seed centered at $(-52,12,0)$ and the temporal pole. The rsFC with the other two seeds did not positively correlate with increased PVFT score in any region. No regions showed negative correlations between the rsFC with any seeds and the increased PVFT score. The regression analysis findings are summarized in Table 3.

Table 3 about here

\section{Discussion}


The present study provides two novel findings. First, significantly higher rsFCs were observed in INT than in CONT between the left inferior frontal gyrus seed and the temporal pole and middle frontal gyrus. Furthermore, the rsFC strength between the left inferior frontal gyrus seed and temporal pole was positively correlated with the increased PVFT score between the pre- and post-intervention periods. Second, significantly lower rsFCs were identified in INT compared to CONT between the left middle frontal gyrus seed and the posterior cingulate cortex, precuneus, and postcentral gyrus. Our findings suggest that the beneficial intervention effect of PICMOR on verbal fluency is related to improved rsFC between the left inferior frontal gyrus and temporal pole. Enhanced rsFC between the left inferior frontal gyrus and middle frontal gyrus and suppressed rsFC between the left middle frontal gyrus and posterior cortical midline structures could also contribute to the enhanced verbal fluency. These findings are discussed further below.

\section{Regions showing higher rsFC with seed regions in INT than in CONT}

Our first major finding was that the rsFCs between the left inferior frontal gyrus as a seed region and the temporal pole and middle frontal gyrus were higher in INT than in CONT (Fig. 1). Significant activation in the temporal pole has been reported in a task-related fMRI study of phonemic verbal fluency [21]. Previous neuroimaging studies have interpreted task-related activation in the anterior temporal lobe, including the temporal pole, in terms of semantic processing [22-24]. This interpretation is supported by evidence from neuropsychological studies of patients with semantic dementia and neurostimulation studies of healthy adults [25-31]. For example, neurostimulation studies using repetitive transcranial magnetic stimulation (rTMS) have consistently shown that the application of rTMS to the left or right temporal pole disrupted semantic processing [32-34]. According to the hub-and-spoke hypothesis, the anterior temporal lobe acts as a hub in semantic processing, which interacts with modality-specific spokes of sensory, motor, and linguistic regions, including the inferior frontal gyrus [26-29]. Supporting this hypothesis, the intrinsic and anatomical connectivity between the anterior temporal lobe and inferior frontal gyrus have been identified in several neuroimaging studies [35-38]. Taken together with previous findings that intrinsic connectivity patterns can predict an individual's ability to perform cognitive tasks [6], the significantly greater rsFC between the left inferior frontal gyrus and temporal pole in INT compared to CONT suggests that enhanced interactive mechanisms between the two regions at rest contribute to the enhanced ability of INT to access their mental lexicon and retrieve words from their semantic memory. Consistent with this, the results of the regression analyses demonstrated that the rsFC strength between the left inferior frontal gyrus and temporal pole positively correlated with the increased PVFT score between the pre- and post-intervention periods.

Significant activation in the middle frontal gyrus has been identified in several task-related fMRI studies using PVFT [39-45]. Activation in the dorsolateral prefrontal cortex has also been observed in several neuroimaging meta-analyses of the N-back task [46], subsequent memory task [47], Go-NoGo task [48, 49], and Stroop task [50]. All these tasks require executive control processes for successful performance, including retaining various items and task rules in the working memory and/or suppressing inappropriate 
responses. Thus, the significantly higher rsFC between the left inferior frontal gyrus and middle frontal gyrus in INT compared to CONT suggests that enhanced interactive mechanisms between these regions during the resting-state contribute to the enhanced executive control ability in word production processes, such as retaining earlier verbal responses in working memory and avoiding repetition.

\section{Regions showing lower rsFC with seed regions in INT than in CONT}

The second major finding was that the rsFCs between the left middle frontal gyrus as a seed region and posterior cortical midline structures were lower in INT than in CONT (Fig. 1). Posterior cortical midline structures, including the posterior cingulate cortex, have been shown to deactivate during PVFT [41, 42, $44,45,51]$. This area has been proposed as a core component of the default mode network [52, 53] (see supplementary file), which shows greater activation during the resting-state than during the performance of attention-demanding cognitive tasks [54-57]. Although the precise role of the default mode network is still a matter of debate, one possible explanation for activation in the default mode network is that it could involve spontaneous thoughts, i.e., mind-wandering, daydreaming, or task-irrelevant thoughts [5860]. Given that successful performance in PVFT may rely on the suppression of task-irrelevant thoughts, it is reasonable to suppose that suppressed interactive mechanisms between the left middle frontal gyrus and the default mode network in INT compared with CONT at resting-state contribute to the enhanced ability of INT to suppress spontaneous thoughts during word production.

Results from the regression analyses showed that there were no significant correlations between the rsFC strength for the left middle frontal gyrus seed with the default mode network and the difference in the PVFT score between the pre- and post-intervention periods. This suggests that the suppressed interactive mechanisms between these regions were not directly associated with the beneficial intervention effect of PICMOR on verbal fluency. This could be interpreted as evidence of increased attentional ability. Taken together with previous findings that anticorrelation of the default mode network with task-positive regions was associated with successful task performance [61, 62], the anticorrelated rsFC between the left middle frontal gyrus and the default mode network might reflect increased attentional ability that could indirectly contribute to the beneficial intervention effect on verbal fluency.

\section{Limitations and future directions}

The present study has several limitations. First, any differences in rsFCs between INT and CONT cannot be entirely attributed to the intervention effects because we lack comparable rsfMRI data from the preintervention period. In this pilot study, we cannot make any definitive conclusions because it is possible that the two groups intrinsically differed in the functional networks of the brain regions involved in verbal fluency even before the intervention. However, taken together with the behavioral evidence, which showed there was no significant difference in the PVFT score between INT and CONT before the intervention (see Table 1), it is unlikely that our present rsfMRI findings reflect such an intrinsic difference in the brain 
network between the groups. To obtain a more definitive conclusion, future research will need to obtain rsfMRI data before and after the intervention.

Second, we cannot accurately identify the components in the PICMOR program that contributed to the beneficial intervention effect on verbal fluency. During group conversations, participants in INT received repeated training to make a speech within a limited time (i.e., 1 min), flexibly ask and answer questions, intentionally store and manipulate information to ask questions, and suppress the interruption of other members. In contrast, those in CONT did not receive such training. Given the difference in training demands to exercise executive functions between the two groups, it is not surprising that a significantly larger enhancement in the PVFT score occurred for INT compared to CONT. However, we cannot identify which components in the training contributed to the beneficial intervention effect of PICMOR on verbal fluency. Further investigation is required to clarify this relationship.

Third, our study may have been limited by selection bias for seed regions. In the present study, we applied a seed-based functional connectivity analysis to rsfMRI data. While this approach enabled us to explore rsFC with seed regions at the whole-brain level, it inevitably resulted in selection bias for the seed regions and, therefore, provides a limited amount of information on rsFC across the whole-brain. However, we had valid reasons for selecting the left inferior and middle frontal gyri as seed regions, i.e., we previously found a significantly larger enhancement in the PVFT score in INT than in CONT [4], and there is strong evidence from neuropsychological and neuroimaging studies that the left inferior and middle frontal gyri play a critical role in verbal fluency [10-15]. Therefore, we believe that the seed-based approach was still an effective way to achieve the aim of the present study. A data-driven approach, including whole-brain multivariate pattern and independent component analyses [63], could be used in future research to overcome the disadvantage of the seed-based approach.

The final limitation is related to the correction for multiple comparisons in the rsfMRI data analysis. Specifically, in the present study, the correction for the whole-brain multiple comparisons was performed using FDR, $p<0.05$. However, because we selected six seed regions to compare INT and CONT, adopting a stricter statistical significance might be more accurate. When we employed the height threshold divided by the number of seed regions in the two-sample $t$-tests, some rsFC values survived, including the rsFCs between the left inferior frontal gyrus seed centered at $(-44,18,6)$ and the middle frontal gyrus, and between the left middle frontal gyrus seed centered at $(-42,8,6)$ and the posterior cingulate cortex. However, other rsFC values failed to achieve stricter statistical significance. Despite this limitation, this preliminary report successfully achieved its exploratory purpose and provided candidates for rsFCs, reflecting the beneficial effects of PICMOR on the brain network responsible for verbal fluency.

In future research, it is important to elucidate the neural mechanisms by which verbal fluency is enhanced by interventions, given that there is some behavioral evidence that verbal fluency can be trained [64]. This pilot study provides a basis for such future research. In our previous RCT, the ability of verbal fluency was assessed by a neuropsychological test in an experimental setting [4], as in other intervention studies [2]. However, little is known about how interventions affect cognitive task performance in real-world 
situations. Future studies that compare verbal fluency in day-to-day conversations between the pre- and post-intervention periods could identify how the intervention effects identified in experimental settings could be extended to real-world situations.

\section{Conclusions}

In the present study, we investigated the differences in the functional networks of the brain regions involved in verbal fluency at resting-state between INT and CONT after the intervention period. Results showed that the rsFC between the left inferior frontal gyrus and the temporal pole was higher in INT than in CONT, and that the rSFC strength was positively correlated with the increased PVFT score between the pre- and post-intervention periods. The rsFC between the left inferior frontal gyrus and the middle frontal gyrus was also higher in INT than in CONT. In contrast, the rsFCs between the left middle frontal gyrus and the posterior cortical midline structures were lower in INT than in CONT. These findings suggest that the beneficial intervention effect of PICMOR on verbal fluency is related to increased rsFC of the left inferior frontal gyrus with the semantic-related temporal pole. The increased rsFC between the left inferior frontal gyrus and executive control-related middle frontal gyrus, as well as the suppressed rsFC between the left middle frontal gyrus and posterior cortical midline structures, could also contribute to the enhancement of verbal fluency. No definitive conclusions can be drawn because our study lacked comparable rsfMRI data from the pre-intervention period. However, this pilot study achieved its exploratory purpose and provided candidates for rsFCs, reflecting the neural mechanisms that underlie the beneficial intervention effect of PICMOR on verbal fluency.

\section{Abbreviations}

PICMOR: Photo-Integrated Conversation Moderated by Robots; RCT: randomized controlled trial; PVFT: phonemic verbal fluency task; INT: the intervention group; CONT: the control group; rsfMRI: resting-state functional magnetic resonance imaging; rsFC: resting-state functional connectivity; TR: repetition time; TE: echo time; FOV: field of view; BOLD: blood oxygenation level-dependent; SPM: Statistical Parametric Mapping; MNI: Montreal Neurological Institute; FDR: false discovery rate; SD: standard deviation; C.I.: confidence interval; rTMS: repetitive transcranial magnetic stimulation

\section{Declarations}

\section{Ethics approval and consent to participate}

The present study was approved by the Institutional Review Board of RIKEN (Wako3 30-11 (2)), and written informed consent was obtained from all participants.

\section{Consent for publication}

Not applicable. 


\section{Availability of data and materials}

A joint research agreement is required for data sharing.

\section{Competing interests}

The authors declare that they have no competing interests.

\section{Funding}

This work was supported by JSPS KAKENHI Grant Numbers JP17H05920 (MO-M), JP18KT0035 (MO-M), JP19H01138 (MO-M), and JP19K14489 (HS). The funding body had no role in the design of the study and collection, analysis, and interpretation of data and in writing the manuscript.

\section{Authors' contributions}

MO-M designed this study. HS collected and analyzed the data and wrote the manuscript under the supervision of TK and MO-M. All authors have read and approved the final manuscript.

\section{Acknowledgments}

The research experiments were conducted using the MRI scanner at the Advanced Imaging Center Yaesu Clinic, Tokyo. We thank the staff for their technical assistance in the MRI scanning. We also thank Drs. Takuya Sekiguchi and Seiki Tokunaga for their advice on improving the manuscript.

\section{References}

1. World Health Organization. Risk reduction of cognitive decline and dementia - WHO Guidelines. Retrieved from https://www.who.int/mental_health/neurology/dementia/guidelines_risk_reduction/en/ (2019).

2. Kelly ME, Duff H, Kelly S, McHugh Power JE, Brennan S, Lawlor BA, et al. The impact of social activities, social networks, social support and social relationships on the cognitive functioning of healthy older adults: a systematic review. Syst Rev. 2017;6(1):259.

3. Livingston G, Sommerlad A, Orgeta V, Costafreda SG, Huntley J, Ames D, et al. Dementia prevention, intervention, and care. Lancet. 2017;390(10113):2673-734.

4. Otake-Matsuura M, Tokunaga S, Watanabe K, Abe MS, Sekiguchi T, Sugimoto H, et al. Photointegrated conversation moderated by robots for cognitive health in older adults: a randomized controlled trial. Preprint at https://doi.org/10.1101/19004796 (2019). 
5. Fujiwara $Y$, Suzuki H, Yasunaga M, Sugiyama M, ljuin M, Sakuma N, et al. Brief screening tool for mild cognitive impairment in older Japanese: validation of the Japanese version of the Montreal Cognitive Assessment. Geriatr Gerontol Int. 2010;10(3):225-32.

6. Harmelech T, Malach R. Neurocognitive biases and the patterns of spontaneous correlations in the human cortex. Trends Cogn Sci. 2013;17(12):606-15.

7. Miro-Padilla A, Bueicheku E, Ventura-Campos N, Palomar-Garcia MA, Avila C. Functional connectivity in resting state as a phonemic fluency ability measure. Neuropsychologia. 2017;97:98-103.

8. Sala-Llonch R, Bartres-Faz D, Junque C. Reorganization of brain networks in aging: a review of functional connectivity studies. Front Psychol. 2015;6:663.

9. Broca P. Remarques sur le siege de la faculte du langage articule; suivies d'une observation d'aphemie (perte de la parole). Bull Mem Soc Anat Paris. 1861;36:330-57.

10. Costafreda SG, Fu CHY, Lee L, Everitt B, Brammer MJ, David AS. A systematic review and quantitative appraisal of $\mathrm{fMRI}$ studies of verbal fluency: role of the left inferior frontal gyrus. Hum Brain Mapp. 2006;27(10):799-810.

11. Wagner S, Sebastian A, Lieb K, Tuscher O, Tadic A. A coordinate-based ALE functional MRI metaanalysis of brain activation during verbal fluency tasks in healthy control subjects. Bmc Neurosci. 2014;15.

12. Baldo JV, Schwartz S, Wilkins D, Dronkers NF. Role of frontal versus temporal cortex in verbal fluency as revealed by voxel-based lesion symptom mapping. J Int Neuropsychol Soc. 2006;12(6):896-900.

13. Baldo JV, Shimamura AP, Delis DC, Kramer J, Kaplan E. Verbal and design fluency in patients with frontal lobe lesions. J Int Neuropsychol Soc. 2001;7(5):586-96.

14. Robinson G, Shallice T, Bozzali M, Cipolotti L. The differing roles of the frontal cortex in fluency tests. Brain. 2012;135(7):2202-14.

15. Stuss DT, Alexander MP, Hamer L, Palumbo C, Dempster R, Binns M, et al. The effects of focal anterior and posterior brain lesions on verbal fluency. J Int Neuropsychol Soc. 1998;4(3):265-78.

16. Shao Z, Janse E, Visser K, Meyer AS. What do verbal fluency tasks measure? Predictors of verbal fluency performance in older adults. Front Psychol. 2014;5:772.

17. Levelt WJ, Roelofs A, Meyer AS. A theory of lexical access in speech production. Behav Brain Sci. 1999;22(1):1-38; discussion 38-75.

18. Ruff RM, Light RH, Parker SB, Levin HS. The psychological construct of word fluency. Brain Lang. 1997;57(3):394-405.

19. Whitfield-Gabrieli S, Nieto-Castanon A. Conn: a functional connectivity toolbox for correlated and anticorrelated brain networks. Brain Connect. 2012;2(3):125-41.

20. Behzadi Y, Restom K, Liau J, Liu TT. A component based noise correction method (CompCor) for BOLD and perfusion based fMRI. Neuroimage. 2007;37(1):90-101.

21. Heim S, Eickhoff SB, Amunts K. Specialisation in Broca's region for semantic, phonological, and syntactic fluency? Neuroimage. 2008;40(3):1362-8. 
22. Binder JR, Desai RH, Graves WW, Conant LL. Where is the semantic system? A critical review and meta-analysis of 120 functional neuroimaging studies. Cereb Cortex. 2009;19(12):2767-96.

23. Rice GE, Lambon Ralph MA, Hoffman P. The roles of left versus right anterior temporal lobes in conceptual knowledge: an ALE meta-analysis of 97 functional neuroimaging studies. Cereb Cortex. 2015;25(11):4374-91.

24. Visser M, Jefferies E, Lambon Ralph MA. Semantic processing in the anterior temporal lobes: a metaanalysis of the functional neuroimaging literature. J Cogn Neurosci. 2010;22(6):1083-94.

25. Bonner MF, Price AR. Where is the anterior temporal lobe and what does it do? J Neurosci. 2013;33(10):4213-5.

26. Lambon Ralph MA, Patterson K. Generalization and differentiation in semantic memory: insights from semantic dementia. Ann N Y Acad Sci. 2008;1124:61-76.

27. Patterson K, Nestor PJ, Rogers TT. Where do you know what you know? The representation of semantic knowledge in the human brain. Nat Rev Neurosci. 2007;8(12):976-87.

28. Ralph MA, Jefferies E, Patterson K, Rogers TT. The neural and computational bases of semantic cognition. Nat Rev Neurosci. 2017;18(1):42-55.

29. Rice GE, Hoffman P, Lambon Ralph MA. Graded specialization within and between the anterior temporal lobes. Ann N Y Acad Sci. 2015;1359:84-97.

30. Rogers TT, Lambon Ralph MA, Garrard P, Bozeat S, McClelland JL, Hodges JR, et al. Structure and deterioration of semantic memory: a neuropsychological and computational investigation. Psychol Rev. 2004;111(1):205-35.

31. Wong C, Gallate J. The function of the anterior temporal lobe: a review of the empirical evidence. Brain Res. 2012;1449:94-116.

32. Lambon Ralph MA, Pobric G, Jefferies E. Conceptual knowledge is underpinned by the temporal pole bilaterally: convergent evidence from rTMS. Cereb Cortex. 2009;19(4):832-8.

33. Pobric G, Jefferies E, Ralph MA. Amodal semantic representations depend on both anterior temporal lobes: evidence from repetitive transcranial magnetic stimulation. Neuropsychologia. 2010;48(5):1336-42.

34. Pobric G, Lambon Ralph MA, Jefferies E. The role of the anterior temporal lobes in the comprehension of concrete and abstract words: rTMS evidence. Cortex. 2009;45(9):1104-10.

35. Fan L, Wang J, Zhang Y, Han W, Yu C, Jiang T. Connectivity-based parcellation of the human temporal pole using diffusion tensor imaging. Cereb Cortex. 2014;24(12):3365-78.

36. Hurley RS, Bonakdarpour B, Wang X, Mesulam MM. Asymmetric connectivity between the anterior temporal lobe and the language network. J Cogn Neurosci. 2015;27(3):464-73.

37. Jackson RL, Hoffman P, Pobric G, Lambon Ralph MA. The semantic network at work and rest: differential connectivity of anterior temporal lobe subregions. J Neurosci. 2016;36(5):1490-501.

38. Papinutto N, Galantucci S, Mandelli ML, Gesierich B, Jovicich J, Caverzasi E, et al. Structural connectivity of the human anterior temporal lobe: a diffusion magnetic resonance imaging study. 
Hum Brain Mapp. 2016;37(6):2210-22.

39. Abrahams S, Goldstein LH, Simmons A, Brammer MJ, Williams SC, Giampietro VP, et al. Functional magnetic resonance imaging of verbal fluency and confrontation naming using compressed image acquisition to permit overt responses. Hum Brain Mapp. 2003;20(1):29-40.

40. Fu CH, Morgan K, Suckling J, Williams SC, Andrew C, Vythelingum GN, et al. A functional magnetic resonance imaging study of overt letter verbal fluency using a clustered acquisition sequence: greater anterior cingulate activation with increased task demand. Neuroimage. 2002;17(2):871-9.

41. Halari R, Sharma T, Hines M, Andrew C, Simmons A, Kumari V. Comparable fMRI activity with differential behavioural performance on mental rotation and overt verbal fluency tasks in healthy men and women. Exp Brain Res. 2006;169(1):1-14.

42. Hutchinson M, Schiffer W, Joseffer S, Liu A, Schlosser R, Dikshit S, et al. Task-specific deactivation patterns in functional magnetic resonance imaging. Magn Reson Imaging. 1999;17(10):1427-36.

43. Meinzer M, Flaisch T, Wilser L, Eulitz C, Rockstroh B, Conway T, et al. Neural signatures of semantic and phonemic fluency in young and old adults. J Cogn Neurosci. 2009;21(10):2007-18.

44. Meinzer M, Seeds L, Flaisch T, Harnish S, Cohen ML, McGregor K, et al. Impact of changed positive and negative task-related brain activity on word-retrieval in aging. Neurobiol Aging. 2012;33(4):65669.

45. Tseng CJ, Froudist-Walsh S, Kroll J, Karolis V, Brittain PJ, Palamin N, et al. Verbal fluency is affected by altered brain lateralization in adults who were born very preterm. eNeuro. 2019;6(2).

46. Owen AM, McMillan KM, Laird AR, Bullmore E. N-back working memory paradigm: a meta-analysis of normative functional neuroimaging studies. Hum Brain Mapp. 2005;25(1):46-59.

47. Blumenfeld RS, Ranganath C. Dorsolateral prefrontal cortex promotes long-term memory formation through its role in working memory organization. J Neurosci. 2006;26(3):916-25.

48. Buchsbaum BR, Greer S, Chang WL, Berman KF. Meta-analysis of neuroimaging studies of the Wisconsin card-sorting task and component processes. Hum Brain Mapp. 2005;25(1):35-45.

49. Steele VR, Aharoni E, Munro GE, Calhoun VD, Nyalakanti P, Stevens MC, et al. A large scale (N=102) functional neuroimaging study of response inhibition in a Go/NoGo task. Behav Brain Res. 2013;256:529-36.

50. Laird AR, McMillan KM, Lancaster JL, Kochunov P, Turkeltaub PE, Pardo JV, et al. A comparison of label-based review and ALE meta-analysis in the Stroop task. Hum Brain Mapp. 2005;25(1):6-21.

51. Schlosser R, Hutchinson M, Joseffer S, Rusinek H, Saarimaki A, Stevenson J, et al. Functional magnetic resonance imaging of human brain activity in a verbal fluency task. J Neurol Neurosurg Psychiatry. 1998;64(4):492-8.

52. Fransson P, Marrelec G. The precuneus/posterior cingulate cortex plays a pivotal role in the default mode network: evidence from a partial correlation network analysis. Neuroimage. 2008;42(3):117884. 
53. Utevsky AV, Smith DV, Huettel SA. Precuneus is a functional core of the default-mode network. J Neurosci. 2014;34(3):932-40.

54. Buckner RL, Andrews-Hanna JR, Schacter DL. The brain's default network: anatomy, function, and relevance to disease. Ann N Y Acad Sci. 2008;1124:1-38.

55. Mak LE, Minuzzi L, MacQueen G, Hall G, Kennedy SH, Milev R. The default mode network in healthy individuals: a systematic review and meta-analysis. Brain Connect. 2017;7(1):25-33.

56. Raichle ME. The brain's default mode network. Annu Rev Neurosci. 2015;38:433-47.

57. Raichle ME, MacLeod AM, Snyder AZ, Powers WJ, Gusnard DA, Shulman GL. A default mode of brain function. Proc Natl Acad Sci U S A. 2001;98(2):676-82.

58. Fox KC, Spreng RN, Ellamil M, Andrews-Hanna JR, Christoff K. The wandering brain: meta-analysis of functional neuroimaging studies of mind-wandering and related spontaneous thought processes. Neuroimage. 2015;111:611-21.

59. Gruberger M, Ben-Simon E, Levkovitz Y, Zangen A, Hendler T. Towards a neuroscience of mindwandering. Front Hum Neurosci. 2011;5:56.

60. Mittner M, Hawkins GE, Boekel W, Forstmann BU. A neural model of mind wandering. Trends Cogn Sci. 2016;20(8):570-78.

61. Esposito R, Cieri F, Chiacchiaretta P, Cera N, Lauriola M, Di Giannantonio M, et al. Modifications in resting state functional anticorrelation between default mode network and dorsal attention network: comparison among young adults, healthy elders and mild cognitive impairment patients. Brain Imaging Behav. 2018;12(1):127-41.

62. Hampson M, Driesen N, Roth JK, Gore JC, Constable RT. Functional connectivity between taskpositive and task-negative brain areas and its relation to working memory performance. Magn Reson Imaging. 2010;28(8):1051-7.

63. van den Heuvel MP, Hulshoff Pol HE. Exploring the brain network: a review on resting-state fMRI functional connectivity. Eur Neuropsychopharmacol. 2010;20(8):519-34.

64. Dodge HH, Zhu J, Mattek N, Bowman M, Ybarra O, Wild K, et al. Web-enabled conversational interactions as a means to improve cognitive functions: results of a 6-week randomized controlled trial. Alzheimers Dement (N Y). 2015;1(1):1-12.

\section{Tables}

Table 1 Participants' characteristics 
INT $(\mathbf{N}=\quad$ CONT $(\mathbf{N}=$

31)

30)

Age (mean \pm SD)

$72.84 \pm 3 . \quad 72.03 \pm 2.7$

45

2

Sex (males : females)

$16: 15$

$13: 17$

Educational level ( $\geq 13$ years $:<13$ years)

$20: 11$

$17: 13$

Amount of talking time in group conversations during the $5000.52 \pm \quad 7627.19 \pm$

intervention period** $(\mathrm{sec})($ mean $\pm \mathrm{SD})$

800.63

3377.06

PVFT score at the pre-intervention (mean $\pm \mathrm{SD}$ )

$12.03 \pm$

$11.13 \pm$

3.61

4.07

PVFT score at the post-intervention** (mean \pm SD)

$13.71 \pm$

$11.07 \pm$

3.55

2.95

Notes: We lack conversational data of one out of 12 group conversations from two CONT groups due to technical errors. Asterisks represent the variables showing significant difference between INT and CONT $(p<0.01)$.

Abbreviations: INT, the intervention group; CONT, the control group; PVFT, phonemic verbal fluency task; SD, standard deviation.

Table 2 Regions showing significant group differences in functional connectivity with seed regions 


\begin{tabular}{|c|c|c|c|c|c|c|c|}
\hline \multirow[t]{2}{*}{ Seed region } & \multirow[t]{2}{*}{ Direction } & \multirow[t]{2}{*}{ Region } & \multicolumn{3}{|c|}{$\begin{array}{c}\text { MNI } \\
\text { coordinates }\end{array}$} & \multirow[t]{2}{*}{ Voxel } & \multirow[t]{2}{*}{$\begin{aligned} \text { Z va } \\
\text { lue }\end{aligned}$} \\
\hline & & & $\mathrm{x}$ & $\mathrm{y}$ & $\mathbf{z}$ & & \\
\hline \multirow[t]{3}{*}{$\begin{array}{l}\text { Left inferior frontal gyrus } \\
(-52,12,0)\end{array}$} & $\begin{array}{r}\text { INT }>\text { CO } \\
\text { NT }\end{array}$ & Temporal pole & 52 & 10 & -42 & 284 & 3.91 \\
\hline & & & 44 & 6 & -40 & & 3.78 \\
\hline & & & 46 & 14 & -38 & & 3.71 \\
\hline \multirow[t]{3}{*}{$\begin{array}{l}\text { Left inferior frontal gyrus } \\
(-44,18,6)\end{array}$} & $\begin{array}{l}\text { INT }> \\
\text { CONT }\end{array}$ & $\begin{array}{l}\text { Middle frontal gyru } \\
\mathrm{s}^{*}\end{array}$ & 50 & 12 & 36 & 404 & 4.23 \\
\hline & & & 46 & 24 & 30 & & 3.90 \\
\hline & & & 54 & 12 & 18 & & 3.81 \\
\hline \multirow[t]{8}{*}{$\begin{array}{l}\text { Left middle frontal gyrus } \\
(-42,8,6)\end{array}$} & $\begin{array}{l}\text { INT }< \\
\text { CONT }\end{array}$ & $\begin{array}{r}\begin{array}{r}\text { Posterior cingulate } \\
\text { cortex* }\end{array}\end{array}$ & -4 & -44 & 10 & 545 & 4.23 \\
\hline & & & 14 & -28 & 4 & & 3.89 \\
\hline & & & -4 & -54 & 2 & & 3.85 \\
\hline & & Precuneus & 0 & -48 & 54 & 162 & 3.77 \\
\hline & & & 4 & -50 & 66 & & 3.57 \\
\hline & & & 6 & -60 & 54 & & 3.19 \\
\hline & & Postcentral gyrus & -10 & -52 & 74 & 179 & 4.87 \\
\hline & & & -6 & -60 & 70 & & 3.92 \\
\hline
\end{tabular}

Notes: Asterisks represent the survived functional connectivity values even when a stricter height threshold was employed to correct for whole-brain multiple comparisons (FDR, $p<$ $0.05 / 6)$.

Abbreviations: INT, the intervention group; CONT, the control group; MNI, Montreal Neurological Institute; FDR, false discovery rate.

Table 3 Regions showing positive correlation between functional connectivity with seed regions and increased PVFT score

\begin{tabular}{lrrrrrr}
\hline Seed region & Region & \multicolumn{3}{c}{ MNI coordinates } & Voxel & Z value \\
\cline { 3 - 5 } & & $\mathbf{x}$ & $\mathbf{y}$ & $\mathbf{z}$ & & \\
\hline Left inferior frontal gyrus $(-52,12,0)$ & Temporal pole & 50 & 6 & -22 & 398 & 4.26 \\
\hline & & 48 & 14 & -22 & 4.12 \\
\hline
\end{tabular}

Abbreviations: PVFT, phonemic verbal fluency task; MNI, Montreal Neurological Institute.

\section{Figures}




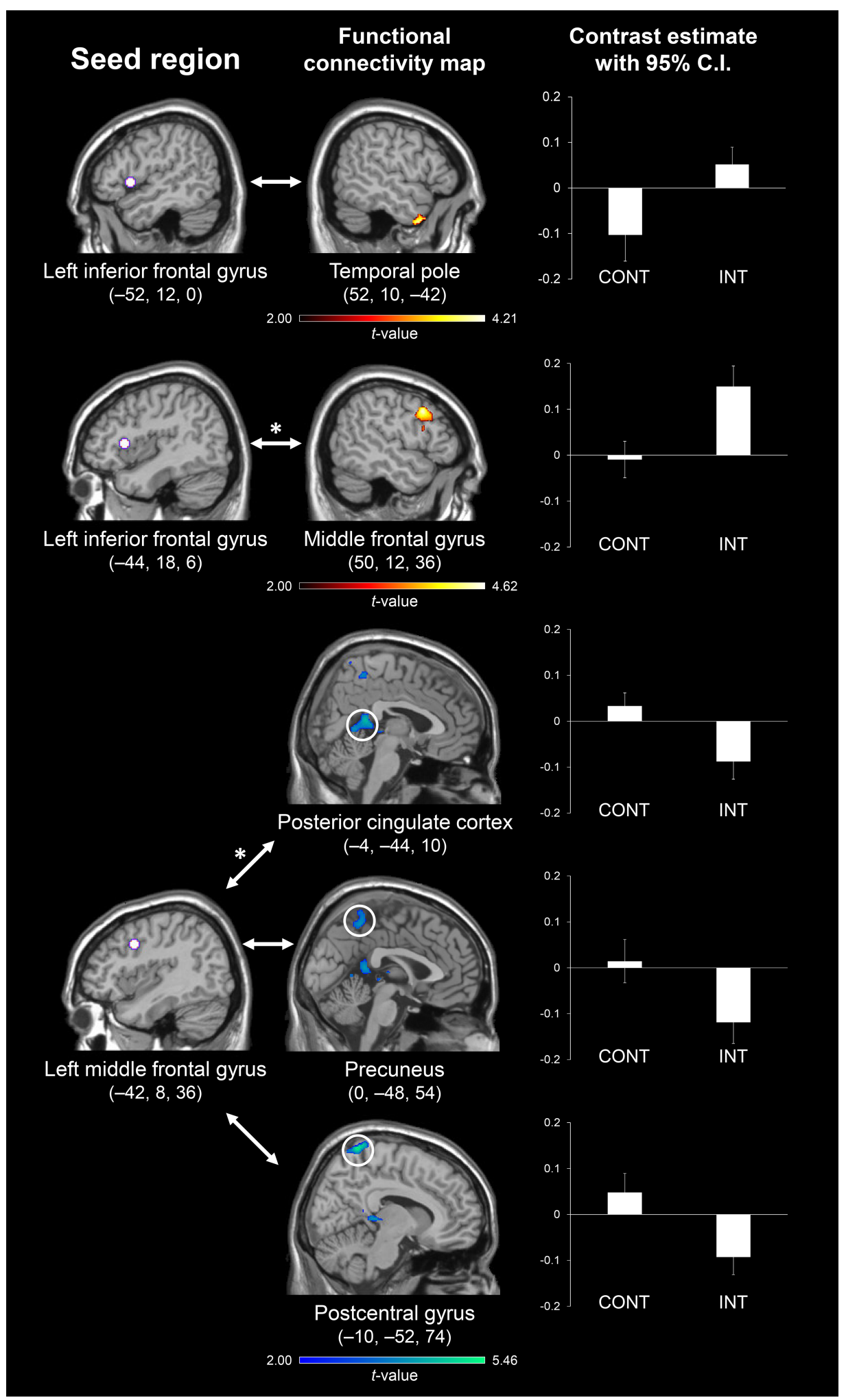

\section{Figure 1}

Functional connectivity showing significant group differences Notes: Regions showing significantly greater rsFC with the left inferior frontal gyrus seed centered at $(-52,12,0)$ and $(-44,18,6)$ in INT than in CONT were identified in the temporal pole and middle frontal gyrus, respectively (red). In contrast, regions showing significantly lower rsFC with the left middle frontal gyrus seed centered at $(-42,8,6)$ in INT than in CONT were identified in the posterior cingulate cortex, precuneus, and postcentral gyrus (blue). The bar 
graphs represent mean contrast estimates within the clusters and error bars represent 95\% C.I. Asterisks indicate the survived functional connectivity values even when a stricter height threshold was adopted to correct for whole-brain multiple comparisons (FDR, $\mathrm{p}<0.05 / 6)$. Abbreviations: rsFC, resting-state functional connectivity; INT, the intervention group; CONT, the control group; C.I., confidence interval; FDR, false discovery rate.

\section{Supplementary Files}

This is a list of supplementary files associated with this preprint. Click to download.

- SugimotoSupplementaryFileBMCGeriatricsrevisedverFinal.docx 